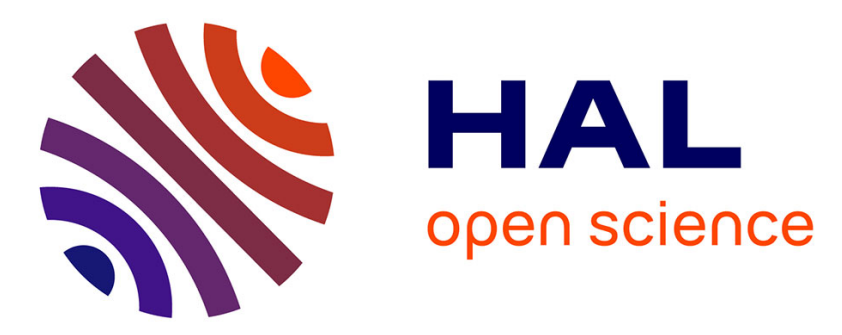

\title{
Transmission and reflection characteristics of metal-coated optical fiber tip pairs
}

Jean-Baptiste Decombe, Jean-François Bryche, Jean-François Motte, Joël

Chevrier, Serge Huant, Jochen Fick

\section{- To cite this version:}

Jean-Baptiste Decombe, Jean-François Bryche, Jean-François Motte, Joël Chevrier, Serge Huant, et al. Transmission and reflection characteristics of metal-coated optical fiber tip pairs. Applied optics, 2013, 52 (26), pp.6620. 10.1364/AO.52.006620 . hal-00859686

\section{HAL Id: hal-00859686 https://hal.science/hal-00859686}

Submitted on 9 Sep 2013

HAL is a multi-disciplinary open access archive for the deposit and dissemination of scientific research documents, whether they are published or not. The documents may come from teaching and research institutions in France or abroad, or from public or private research centers.
L'archive ouverte pluridisciplinaire HAL, est destinée au dépôt et à la diffusion de documents scientifiques de niveau recherche, publiés ou non, émanant des établissements d'enseignement et de recherche français ou étrangers, des laboratoires publics ou privés. 


\title{
Transmission and reflection characteristics of metal-coated optical fiber tip pairs
}

\author{
Jean-Baptiste Decombe, Jean-François Bryche, Jean-François Motte, Joël Chevrier, \\ Serge Huant, and Jochen Fick* \\ Institut Néel, CNRS \& Université Joseph Fourier, 25 Avenue des Martyrs, 38042 Grenoble, France \\ ${ }^{*}$ Corresponding author: jochen.fick@grenoble.cnrs.fr
}

Received 13 June 2013; revised 26 July 2013; accepted 29 July 2013;

posted 1 August 2013 (Doc. ID 192210); published 9 September 2013

\begin{abstract}
The optical transmission and reflection in between two metalized optical fiber tips is studied in the optical near-field and far-field domains. In addition to aluminum-coated tips for near-field scanning optical microscopy (NSOM), specifically developed gold-coated fiber tips cut by focused ion beam are investigated. Transverse transmission maps of subwavelength width clearly indicate optical near-field coupling between the tips for short tip distances and become essentially Gaussian-shaped for larger distances in the far-field regime. Moreover, concentric reflection fringes observed for NSOM-type tips illustrate the influence of the receiving fiber tip on the emission pattern of the source tip. () 2013 Optical Society of America

OCIS codes: $\quad$ (350.3950) Micro-optics; (350.4238) Nanophotonics and photonic crystals; (180.4243)

Near-field microscopy.

http://dx.doi.org/10.1364/AO.52.006620
\end{abstract}

\section{Introduction}

Optical fiber conical tips with a subwavelength clear aperture at the apex are common tools in micro- and nano-optics. They are used for optical trapping of microparticles in single-fiber-tip [1-3] and counterpropagating two-fiber-tip $[\underline{4}, \underline{5}]$ configurations. Fiber tips are also used in scanning optical microscopy: bare fiber tips can be applied for imaging of neurons [6], whereas metalized tips are the key element in near-field scanning optical microscopy (NSOM) $[7,8]$.

The optical near field of metalized fiber tips was probed by using fluorescent nanospheres, and an analytical model for the emitted electric field was developed [9]. The near-field intensity shows two lobes, whereas the far-field emission is polarization dependent and of excellent Gaussian shape with large emission angles exceeding $90^{\circ}$ in the $P$ polarization $[\underline{8}, \underline{10}$. The emission angle can be directly linked to

$1559-128 \mathrm{X} / 13 / 266620-06 \$ 15.00 / 0$

(C) 2013 Optical Society of America the tip apex size. These features have been essentially recovered by finite-difference time-domain calculations [11]. To some extent, a subwavelength aperture at the apex of a conical tip mimics a single small diffraction hole on a flat metallic screen, a problem that has been extensively studied $[\underline{12}, \underline{13}]$ since the pioneer work of Bethe [14].

Recently the power propagation in apertureless metal-coated optical fiber tips was investigated theoretically [15], and tip-to-tip scans of such fiber tips were studied in view of their applications to NSOM lithography [16].

Two open-aperture metalized fiber tips facing each other at a distance of some hundred nanometers are a promising approach for optical nanotweezers. They combine the advantages of nanotraps based on a plasmonic cavity $[17,18]$ with the flexibility of fiber-based optical tweezers [3]. This makes it possible to realize genuine plasmonic tweezers, allowing not only the trapping of nanoparticles but also their manipulation at the nanoscale, with the rewarding prospect of possible operation in air, not only in a liquid. 
In this paper we present the optical transmission and reflection study of metalized fiber tip pairs with tip distances between tens of nanometers and tens of micrometers, thus covering the optical near-field and far-field ranges. More specifically, the transition between these two regimes is studied. Such tip pairs are aimed at being used in future near-field optical tweezers.

\section{Experimental Details}

Two different types of metalized fiber tip are studied: NSOM tips and focused-ion-beam (FIB)-cut tips. The first type consists of open aperture, aluminumcoated fiber tips currently used for NSOM. The second type has gold-coated fiber tips, where the aperture is obtained by FIB-cutting of the entirely metalized fiber tips. The main difference between these two tip types consists in the shape of the tip apex. The application of two different metals does not influence the results presented in this paper.

The elaboration of both tip types is based on chemical wet etching of single-mode, pure silica core fibers (Nufern S630-HP) [19]. The obtained fiber tip angle is about $15^{\circ}$. In the case of NSOM tips, a magnesium fluoride $\left(\mathrm{MgF}_{2}\right)$ film is first deposited on the asetched tips in order to control the final apex diameter. Then an opaque aluminum film of about $100 \mathrm{~nm}$ thickness is deposited by thermal evaporation after the deposition of a thin nickel-chromium adhesion layer. This technique allows one to obtain apex diameters of the order of 200-300 nm without any further step, such as FIB cutting [Fig. 1(a)].

Cutting the fiber tips using FIB leads to smooth, high-quality end faces [Fig. 1(b)]. This point is of paramount importance for experiments with tip distances in the nanometer regime, thus justifying the more complex fabrication process compared to the NSOM tips. At the same time we start using gold for its capacity to support low-loss surface plasmons. Aiming at the smallest achievable aperture sizes, we dismiss the thick $\mathrm{MgF}_{2}$ layer. The $200 \mathrm{~nm}$ gold layer is deposited directly on the etched fiber tips, using only a $10 \mathrm{~nm}$ titanium adhesion layer. The fiber tip end is then cut by FIB to get submicrometer tip apertures. With this technique the obtained tips are of elliptical or nearly circular shape, thus allowing us to study shape effects.

The transmission and reflection of optical fibers are measured on a dedicated setup allowing scanning the relative fiber position with nanometer accuracy (Fig. 2). One fiber is mounted on a set of $x y z$ piezoelectric translation stages (PI P620) with subnanometer resolution and $50 \mu \mathrm{m}$ range. The second fiber is mounted on three perpendicular inertial piezoelectric translation stages (Mechonics MS 30) with $\approx 30 \mathrm{~nm}$ step size and up to $2.5 \mathrm{~cm}$ range. A microscope with a long working distance objective (Mitutoyo M 50x) coupled to a CMOS camera allows the fiber tips to be visualized with micrometer resolution.
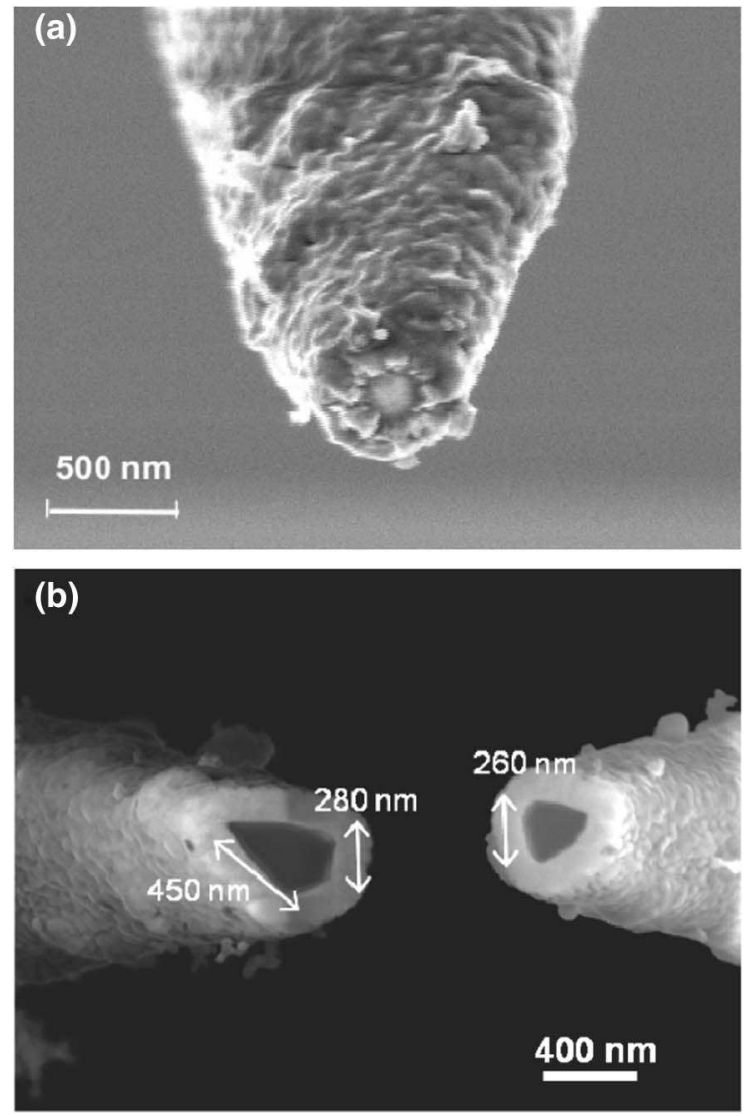

Fig. 1. Scanning electron micrographs of the two fiber tip types used: (a) typical NSOM tip and (b) the FIB-cut tips used in the experiments.

For technical reasons we use two different diode lasers emitting at 808 and $830 \mathrm{~nm}$ to characterize the aluminum- and gold-coated fiber tips, respectively. The small wavelength difference has no influence on the results, as the wavelengths are far away from metal absorption bands or fiber cutoff wavelengths. A 50/50 waveguide coupler is used to allow simultaneous reflection and transmission measurements by means of two amplified silicon photodiodes (New Focus 2001-FC).

A closed-loop control is implemented to stabilize the relative fiber tip distance at the nanometer scale. In fact, thermal drifts are a serious issue in a room temperature uncontrolled environment. The axial

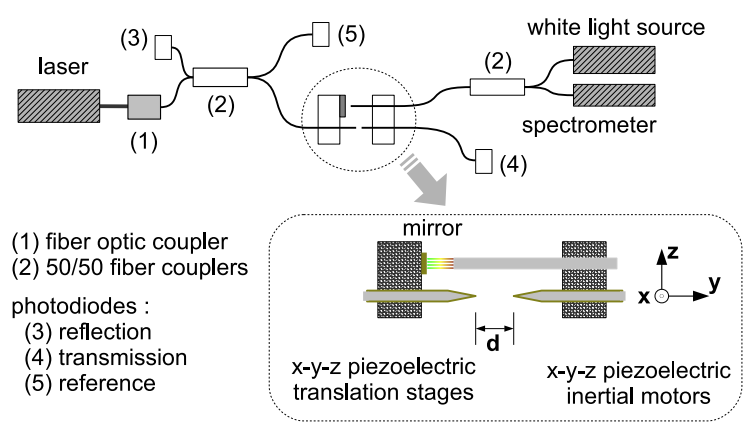

Fig. 2. Scheme of the experimental setup. 
fiber tip distance is the most critical one, as the transverse position can be calibrated by using the transmission maximum position. The feedback signal is obtained from a Fabry-Perot cavity built by a cleaved optical fiber and a metallic mirror, separately mounted on the two fiber tip holders (Fig. 2). A fiber-coupled white lamp source and a minispectrometer (Avantes) allow us to measure the reflection spectra of the Fabry-Perot cavity. Its Fourier transform directly gives the absolute cavity size.

Using the closed-loop control, the relative fiber tip distance can be controlled with a precision better than $\pm 5 \mathrm{~nm}$ over more than $5 \mathrm{~h}$. The absolute tip distance is, however, more difficult to assess. The only accurate way is to perform transverse scans with decreasing distance until the tips are touching. This contact can be clearly observed on the microscope image or by the appearance of streaks in the transmission intensity plot. However, the fiber tip fragility may result in severe damage. After observation of a number of contact events, we estimate that the absolute distance can be determined from the microscope images with $50-100 \mathrm{~nm}$ precision. As a consequence, the minimal distance of the transverse scans used in Section 3 to determinate $w_{0}$ is of the same range.

Control software in the LabView environment allows controlling the entire setup and recording the intensity maps. Transverse transmission and reflection maps for constant distance $d$ are recorded by scanning one fiber in a plane perpendicular to the fiber tips' orientation. Typical scans contain $75 \times 75$ data points. The intensity is averaged over 500 points with read-out frequency of $10 \mathrm{kHz}$ and a photodiode internal amplification of 50-70 dB.

The obtained intensity plots are fitted to the Gaussian intensity beam profile function

$$
I(r)=I_{0} \cdot e^{\frac{-2\left(r-r_{0}\right)^{2}}{w^{2}}}
$$

with $I_{0}$ the intensity amplitude, $r_{0}$ the beam position, and $w$ the beam waist.

The recorded transmission maps correspond to the convolution of the emission and the capture functions of the two fiber tips. Thus, in order to deduce the emission spot width of only one fiber, the measured transmission width has to be corrected. The corresponding deconvolution of two Gaussian functions can be done with

$$
\tilde{w}=\sqrt{w^{2}-w_{0}^{2}}
$$

with $\tilde{w}$ and $w$ the corrected and measured waists, respectively. $w_{0}$ is the optical aperture size of the second fiber tip. For two identical tips $w_{0}$ can be obtained by $w_{0}=w^{\min } / \sqrt{2}, w^{\min }$ being the measured spot size at smallest tip distances.

\section{Results and Discussion}

\section{A. Near-Field Scanning Optical Microscopy Fiber Tips}

Transmission and reflection maps of NSOM fiber tips are recorded for tip distances up to $30 \mu \mathrm{m}$. Typical results are shown in Fig. 3. The transmission spots are of slightly elliptical shape, in agreement with the imperfect circular symmetry of the fiber tips. The minimal measured waist is $w^{\mathrm{min}}=$ $375 \mathrm{~nm}$, corresponding to a corrected waist of $\tilde{w}^{\min }=265 \mathrm{~nm}$, about one-third of the wavelength $\lambda / 3=269 \mathrm{~nm}$. The apex sizes determined by scanning electron microscopy of the two applied tips are 276 and $246 \mathrm{~nm}$. The size of the transmission spot is thus clearly determined by the tip apex and not by the actual wavelength.

The transmission spot size increases linearly with the tip distance for distances greater than $\approx 1 \mu \mathrm{m}$. The corresponding emission angle is $\theta=18.5^{\circ}$, a value found independently of the fiber tip pairs.

Clear circular and concentric reflection fringes are observed for tip distances up to several micrometers (Fig. 3). The fringe center position coincides with that of the transmission peak maximum. As expected for interference fringes, intensity minima and maxima are observed for

$$
\begin{aligned}
d_{m}^{\min } & =\left(\frac{m}{2}+\frac{1}{4}\right) \cdot \lambda, \\
d_{m}^{\max } & =\frac{m}{2} \cdot \lambda
\end{aligned}
$$

with $\lambda$ the wavelength and $m=1,2, \ldots$ a positive integer.

The circular fringes can be explained by backreflection at the fiber tips. The experimental radii of the fringe minima and maxima are determined by
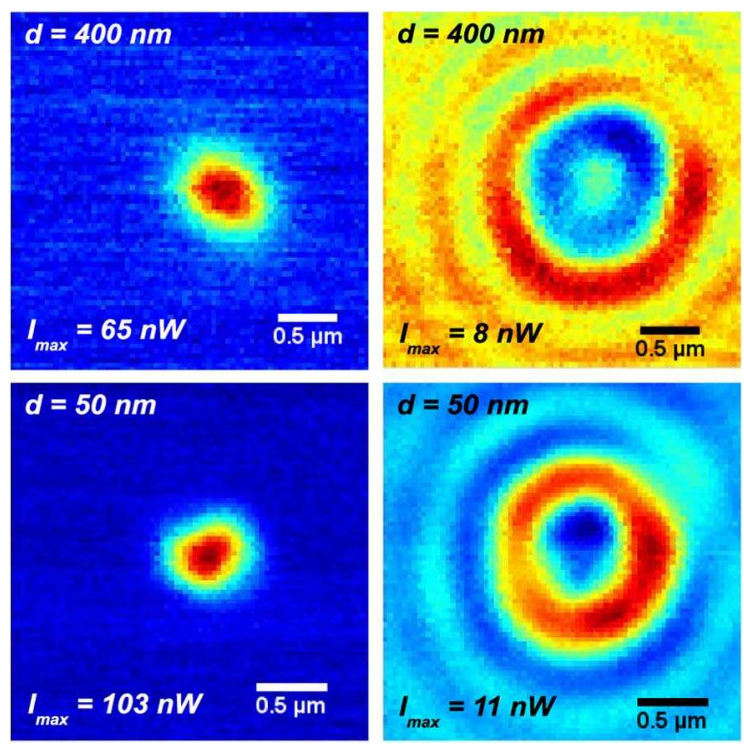

Fig. 3. Transverse transmission (left) and reflection (right) intensity maps of a NSOM tip pair at two distances $d$. The maximal intensities are indicated for an injected power of $P_{\text {in }}=300 \mu \mathrm{W}$. 


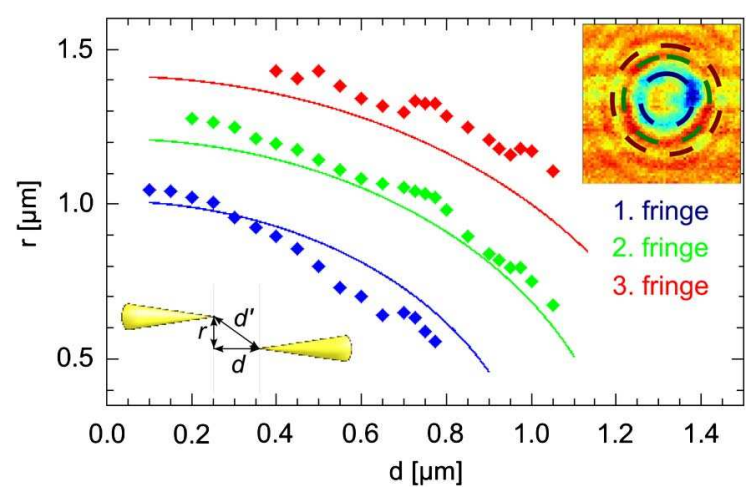

Fig. 4. Position of the interference rings as a function of the axial $(d)$ and transverse $(r)$ fiber tips distances.

fitting concentric circles to the transverse reflection intensity maps. These radii are plotted in Fig. 4 as a function of the two fiber tip planes distance $\bar{d}$. The theoretical fringe positions (lines in Fig. 4) are calculated by using Eq. (3) by replacing $\bar{d}$ with $d^{\prime}=\sqrt{d^{2}+r^{2}}$. The agreement with the experimental values is very good. The observed difference can be attributed to the real (elliptical) shape of the metalized fiber tips.

The observation of distinct reflection patterns clearly shows that the emission pattern of the source fiber tip is influenced by the receiving fiber tip. The reflected intensity is about 1 order of magnitude smaller than the transmitted intensity. However, no fringe is visible on the transmission maps.

\section{B. Focused-Ion-Beam-Cut Fiber Tips}

Now, two FIB-cut fiber tips with different apex shapes are studied. The emission fiber tip is strongly elliptical with major and minor axis diameters of $a=$ 450 and $b=280 \mathrm{~nm}$, respectively [Fig. 1(b), left side]. The receiving tip has a nearly equilateral triangle shape with $260 \mathrm{~nm}$ side length. The measured transmission maps are elliptical for small tip distances and become circular for larger distances (Fig. 5). The elliptical transmission spots are described $\bar{b} y$ two waists $\left(w_{a}\right.$ and $\left.w_{b}\right)$ measured parallel to the major axis $a$ and minor axis $b$, respectively. The as-measured minimal waists of the transmission spot are $w_{a}^{\min }=470 \mathrm{~nm}$ and $w_{b}^{\min }=310 \mathrm{~nm}$.

The injected optical intensity inside the source fiber is $1 \mathrm{~mW}$. The emitted intensity of the source fiber, measured by means of a powermeter, is $1 \mu \mathrm{W}$. The transmitted maximal intensity is 1 and $0.48 \mathrm{nW}$ for tip distances of 100 and $500 \mathrm{~nm}$, respectively. The emission losses of $30 \mathrm{~dB}$ are essentially due to strong propagation losses in the subwavelength wide section near the fiber tip end. For the measurement at $d=100 \mathrm{~nm}$, the reception losses are of the same order as the emission losses, suggesting that the propagation losses between the two fiber tips can be neglected. The lower maximal transmitted intensity of the $d=500 \mathrm{~nm}$ measurements scales with the inverse of the respective transmission spot surfaces.
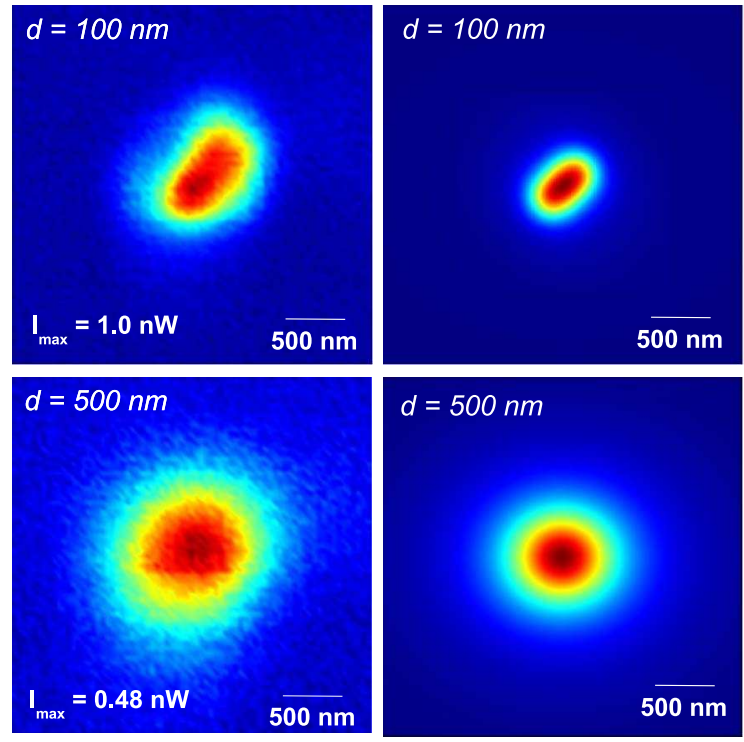

Fig. 5. Experimental (left) and theoretical (right) transverse transmission intensity maps of the FIB-cut fiber tip pair shown in Fig. 1(b). Maximal intensities are indicated for the experimental results.

This means that the totally transmitted optical power is constant.

In contrast to the NSOM tip measurements, no reflection is observed for the FIB-cut tips. This difference originates from the actual shape of the tip apex and not from the different metals used for tip coating. At $808 \mathrm{~nm}$ wavelength the gold and aluminum reflectance is $R^{\mathrm{Au}}=0.976$ and $R^{\mathrm{Al}}=0.867$, respectively. The back-reflection of the gold-coated FIB-cut tips should thus even be slightly more intense than for the aluminum-coated NSOM tips. However, the NSOM tips show an irregular surface with bent edges, whereas the surface of the FIB-cut tips is flat with sharp edges. Therefore, efficient reflection by the FIB-cut tips would require very good parallel alignment of the fiber tips. Moreover reflection could only occur for transverse distances below the actual tip apex size.

The emission of the FIB-cut fiber tips is calculated by using a straightforward electromagnetic model. This model neglects the influence of the reception tip on the field distribution, but allows the main experimental observations to be reproduced. The emitting fiber tip is approximated by an apex of elliptical shape. The optical intensity inside the apex is supposed to be uniform and is represented by a homogeneous distribution of (typically 1164) coherent and orthogonal electric $\mathbf{p}$ and magnetic $\mathbf{m}$ dipole pairs calculated from the incident electromagnetic field $\left(\mathbf{E}^{i}, \mathbf{H}^{i}\right)$ :

$$
\begin{aligned}
\mathbf{p} & =\frac{i}{2 \pi c} \hat{\mathbf{k}} \times \mathbf{H}^{i}, \\
\mathbf{m} & =-\frac{i}{2 \pi c} \hat{\mathbf{k}} \times \mathbf{E}^{i}
\end{aligned}
$$


with $\hat{\mathbf{k}}=\mathbf{E}^{i} \times \mathbf{H}^{i}$ the normalized wave vector and $c$ the speed of light. The emitted electrical field of one single dipole pair is given by [20]

$$
\begin{aligned}
\mathbf{E}(r)= & \frac{1}{4 \pi \epsilon_{0}}\left\{(\hat{\mathbf{r}} \times \mathbf{p}) \times \hat{\mathbf{r}} \frac{k^{2}}{r}+[3 \hat{\mathbf{r}}(\hat{\mathbf{r}} \cdot \mathbf{p})-\mathbf{p}]\left(\frac{1}{r^{3}}-\frac{i k}{r^{2}}\right)\right. \\
& \left.-\frac{1}{Z_{0}}(\hat{\mathbf{r}} \times \mathbf{m}) k^{2}\left(\frac{1}{r}-\frac{1}{i k r^{2}}\right)\right\} e^{i k r}
\end{aligned}
$$

with $\epsilon_{0}$ the vacuum permittivity, $Z_{0}$ the free space impedance, $k=2 \pi / \lambda$ the free space wave vector, and $r$ the distance vector from the dipole. Equation (5) takes all dipole emission terms into account, i.e., nearand far-field contributions. The optical intensity distribution is obtained by summing the contributions over all dipole pairs and squaring the electrical field.

The measured transmission plots correspond to the intensity emission of one tip captured by the second fiber tip. The finite apex of the second tip results in an enlargement of the transmission spot and has to be taken into account. In the present case of two different fiber tips the size correction is less straightforward than for two identical tips. Our results for the aluminum-coated tips show, however, that the minimal waist is of the same order as the tip apex. For correction, the triangular fiber tip is thus approximated by a circular tip of equal surface. Consequently, the calculated emission intensity plots in Fig. $\underline{5}$ are convoluted by a two-dimensional Gaussian function with a waist of $200 \mathrm{~nm}$.

The agreement of the experimental and theoretical transmission maps is satisfactory, and the elliptical and nearly round shapes at respectively small and far distances are well reproduced. The corrected major and minor axis waists $\left(\tilde{w}_{a, b}\right)$ of the elliptical transmission spots are represented in Fig. $\underline{6}$. Here the experimental waists are corrected for the $200 \mathrm{~nm}$ apex size of the second fiber tip. The agreement of observed and calculated values is good for small tip distances and the main features are well reproduced. However, for larger tip distances the calculated values diverge from the experiment. This is

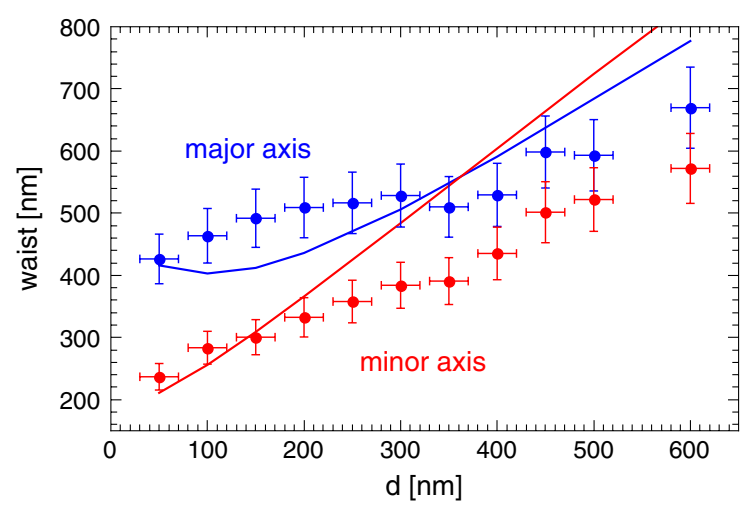

Fig. 6. Major and minor axis transmission waists of the elliptical transmission spot (see Fig. 5) as a function of the tip distance $d$ (points, corrected experimental data with error bars; solid lines, theory). due mainly to very low experimental signal levels and limits of the approximations concerning the simplified apex shapes. Also, it would be interesting to quantify the influence on the field distribution of the receiving tip, which will require full electromagnetic calculations. This is left for future work.

The minimal corrected emission waists of the elliptical fiber tip are $\tilde{w}_{a}^{\min }=428 \mathrm{~nm}$ and $\tilde{w}_{b}^{\min }=$ $243 \mathrm{~nm}$, slightly lower than the actual aperture of the elliptical fiber. At small distances light is transmitted by the optical near field. Thus the shape of the two tip apexes determines the shape of the observed intensity spot. For larger distances the light is transmitted by the optical far field, which cannot resolve the fiber tip shapes smaller than the diffraction limit. Thus, the obtained image corresponds to a point-like optical emitter [21]. This was experimentally observed by the decreasing difference between the major and minor axis waists. The numerical results even show an inversion of the ellipse major and minor axis at $365 \mathrm{~nm}$, slightly below half the wavelength.

\section{Conclusions}

In conclusion, the transmission and reflection properties of two kinds of metal-coated optical fiber tip pairs were experimentally studied. Clear evidence of optical near-field coupling between the two tips of a pair was described by subwavelength transverse transmission spots. This point was confirmed by the resolution of the subwavelength fiber tip shape. We believe that these results are of interest for the future application in optical nanotweezers of this kind of metal-coated optical fiber tip pair.

Funding for this project was provided by a grant from la Région Rhônes-Alpes and by the French National Research Agency in the framework of the FiPlaNT project (ANR-12-BS10-002). Helpful discussions with A. Drezet are gratefully acknowledged.

\section{References}

1. S. Skelton, M. Sergides, R. Patel, E. Karczewska, O. Maragó, and P. Jones, "Evanescent wave optical trapping and transport of micro- and nanoparticles on tapered optical fibers,” J. Quant. Spectrosc. Radiat. Transfer 113, 2512-2520 (2012).

2. S. Eom, Y. Takaya, and T. Hayashi, "Novel contact probing method using single fiber optical trapping probe," Precis. Eng. 33, 235-242 (2009).

3. Z. Liu, C. Guo, J. Yang, and L. Yuan, "Tapered fiber optical tweezers for microscopic particle trapping: fabrication and application," Opt. Express 14, 12510-12516 (2006).

4. S. Valkai, L. Oroszi, and P. Ormos, "Optical tweezers with tips grown at the end of fibers by photopolymerization," Appl. Opt. 48, 2880-2883 (2009).

5. E. R. Lyons and G. J. Sonek, "Confinement and bistability in a tapered hemispherically lensed optical fiber trap,” Appl. Phys. Lett. 66, 1584-1586 (1995).

6. J.-B. Decombe, W. Schwartz, C. Villard, H. Guillou, J. Chevrier, S. Huant, and J. Fick, "A fibered interference scanning optical microscope for living cell imaging," Opt. Express 19, 2702-2710 (2011)

7. E. Betzig, J. Trautman, T. Harris, J. Weiner, and R. Kostelak, "Breaking the diffraction barrier: optical microscopy on a nanometric scale," Science 251, 1468-1470 (1991). 
8. C. Obermüller and K. Karrai, "Far field characterization of diffracting circular apertures," Appl. Phys. Lett. 67, 3408-3410 (1995).

9. A. Drezet, M. Nasse, S. Huant, and J. Woehl, "The optical nearfield of an aperture tip," Europhys. Lett. 66, 41-47 (2004).

10. A. Drezet, J. C. Woehl, and S. Huant, "Diffraction by a small aperture in conical geometry: application to metal-coated tips used in near-field scanning optical microscopy," Phys. Rev. E 65, 046611 (2002).

11. T. J. Antosiewicz and T. Szoplik, "Description of near- and far-field light emitted from a metal-coated tapered fiber tip," Opt. Express 15, 7845-7852 (2007).

12. J.-M. Yi, A. Cuche, F. de Leon-Perez, A. Degiron, E. Laux, C. Genet, J. Alegret, L. Martin-Moreno, and T. Ebbesen, "Diffraction regimes of single holes," Phys. Rev. Lett. 109, 023901 (2012),

13. H. Kihm, S. Koo, Q. Kim, K. Bao, J. Kihm, W. Bak, S. Eah, C. Lienau, H. Kim, P. Nordlander, N. Halas, N. Park, and D.-S. Kim, "Bethe-hole polarization analyser for the magnetic vector of light," Nat. Commun. 2, 451 (2011).

14. H. A. Bethe, "Theory of diffraction by small holes," Phys. Rev. 66, 163-182 (1944).
15. J. Barthes, G. Colas des Francs, A. Bouhelier, and A. Dereux, "A coupled lossy local-mode theory description of a plasmonic tip," New J. Phys. 14, 083041 (2012).

16. I. Kubicova, D. Pudis, L. Suslik, and J. Skriniarova, "Spatial resolution of apertureless metal-coated fiber tip for NSOM lithography determined by tip-to tip scan," Optik 124, 1971-1973 (2013).

17. Y. Pang and R. Gordon, "Optical trapping of a single protein," Nano Lett. 12, 402-406 (2012).

18. Y. Tanaka, S. Kaneda, and K. Sasaki, "Nanostructured potential of optical trapping using a plasmonic nanoblock pair," Nano Lett. 13, 2146-2150 (2013).

19. N. Chevalier, Y. Sonnefraud, J. F. Motte, S. Huant, and K. Karrai, "Aperture-size-controlled optical fiber tips for high-resolution optical microscopy," Rev. Sci. Instrum. 77, 063704 (2006).

20. J. D. Jackson, Classical Electrodynamics, 3rd ed. (Wiley, 1998), Chap. 9.2

21. A. Drezet, A. Cuche, and S. Huant, "Near-field microscopy with a single-photon point-like emitter: resolution versus the aperture tip?" Opt. Commun. 284, 1444-1450 (2011). 